\title{
Inflammation and Insulin Resistance in a Group of Sub-Saharan African Women with Polycystic Ovary Syndrome
}

Christelle Chemaga Nkonpawa (iD) ${ }^{\prime}$ Vicky Jocelyne Ama Moor ${ }^{1,2}$

Aurel T Tankeu ${ }^{3}$

Audrey Synthia Momo'

Guy Sadeu Wafeu ${ }^{4}$

Falmata Amazia ${ }^{2}$

Jan Rene Nkeck (D) ${ }^{2,5}$

Tasha Manases ${ }^{6}$

Julius Dohbit Sama ${ }^{7}$

Simeon Pierre Choukem ${ }^{6,8,9}$

'Department of Biochemistry, Faculty of Medicine and Biomedical Sciences, University of Yaoundé I, Yaoundé,

Cameroon; ${ }^{2}$ Laboratory of Biochemistry, University Teaching Hospital, Yaoundé,

Cameroon; ${ }^{3}$ Department of Biomedical

Sciences, Faculty of Biology and Medicine,

University of Lausanne, Lausanne,

Switzerland; ${ }^{4}$ Centre for Research on

Filariasis and Other Tropical Diseases,

Yaoundé, Cameroon; ${ }^{5}$ Department of

Internal Medicine and Specialties, Faculty

of Medicine and Biomedical Sciences,

University of Yaoundé I, Yaoundé,

Cameroon; ${ }^{6} \mathrm{Health}$ and Human

Development Research Network,

Douala, Cameroon; ${ }^{7}$ Department of

Obstetrics and Gynecology, Faculty of

Medicine and Biomedical Sciences,

University of Yaoundé I, Yaoundé,

Cameroon; ${ }^{8}$ Department of Internal

Medicine and Specialties, Faculty of

Medicine and Pharmaceutical Sciences,

University of Dschang, Dschang,

Cameroon; ${ }^{9}$ Department of Internal

Medicine and Specialties, Douala General

Hospital, Douala, Cameroon

Correspondence: Aurel T Tankeu

Route d'Oron 77, Lausanne, 1010,

Switzerland

Tel +4I76 7723001

Email aurelnet2014@yahoo.com
Background: Studies report high levels of inflammatory markers in women with polycystic ovary syndrome (PCOS), reflecting chronic low-grade inflammation. This inflammation is thought to be associated with insulin resistance. We aim to evaluate inflammatory markers [high sensitivity $\mathrm{C}$ reactive protein (CRP) and interleukin 6] and insulin resistance in women with PCOS in Yaoundé, Cameroon.

Methods: We conducted a comparative cross-sectional study including 32 women with PCOS aged between 18 and 44 years and 32 controls matched for age and body mass index (BMI). Homeostasis model assessment of insulin resistance (HOMA-IR) index calculated using $\mathrm{C}$ peptide levels was used to evaluate insulin resistance. Serum levels of high sensitivity CRP (hsCRP) and interleukin 6 (IL-6) were measured. Comparisons were made using the Student's $T$-test and non-parametric tests (Mann-Whitney $U$-test, Kruskal-Wallis test).

Results: We found that the median [25th-75th percentile] level of hsCRP was significantly higher in women with PCOS compared to the controls $(0.63[0.32-3.81] \mathrm{mg} / \mathrm{L}$ vs. 0.47 [0.15-1.04] mg/L; $p=0.01)$, while IL-6 levels were not different $(8.61[4.1-33.79] \mathrm{pg} / \mathrm{mL}$ for PCOS vs. 8.80 [5.28-38.85] pg/mL for controls; $p=0.51)$. We noted that women with PCOS had a higher HOMA-IR index $(1.15 \pm 0.90$ vs. $0.77 \pm 0.38 ; p=0.03)$. However, there was no correlation between hsCRP level and the HOMA-IR index (Spearman correlation coefficient $=0.10 ; p=0.62$ ).

Conclusion: PCOS is associated with an increased level of hsCRP and insulin resistance in Cameroonian women. This exploratory study provides baseline evidence for larger-scale studies.

Keywords: interleukin 6, high sensitivity $\mathrm{C}$ reactive protein, polycystic ovary syndrome, insulin resistance, sub-Saharan Africa

\section{Introduction}

Polycystic ovary syndrome (PCOS) is the most common endocrinopathy in women of reproductive age. ${ }^{1}$ It is a complex and heterogeneous condition affecting about $10 \%$ of women of childbearing age. ${ }^{2}$ Historically known for its association with poor reproductive health, it has evolved during the past decades as a major background for cardiometabolic diseases. ${ }^{3,4}$ As high as $50-70 \%$ of women with PCOS exhibit insulin resistance, half of them are overweight or obese and present with a 5-7-fold increased risk of type 2 diabetes. $^{3,5}$ Furthermore, large observational studies suggest that women with PCOS might be at a higher risk for developing cardiovascular diseases. ${ }^{6}$ Studies have confirmed low-grade chronic inflammation 
as a key player underlying metabolic syndrome and cardiovascular complications in chronic diseases. ${ }^{7,8}$ Given that, studying low-grade chronic inflammation in PCOS is important as it could be involved in its pathophysiology. Available data provide evidence of a low-grade inflammation with an increased level of hsCRP and IL-6, markers of inflammation in different populations of women with PCOS. ${ }^{9-12}$ However, evidence of inflammation in PCOS is yet to be proven in all populations, and little is known, especially in sub-Saharan African populations who have different clinical and phenotypic presentations. To fill this knowledge gap, we carried out this study to evaluate inflammatory markers in relationship with insulin resistance in Cameroonian women with PCOS, a micro-ecosystem of sub-Saharan Africa.

\section{Materials and Methods Design and Participants}

This comparative cross-sectional study was conducted from March 2020 to June 2020 at the Yaoundé GynecoObstetric and Pediatric Hospital, and at the laboratory of the Yaoundé Central Hospital. We included women aged from 18 to 44 years with PCOS, and control subjects matched for age and BMI. The diagnosis of PCOS was established based on medical records of participants. The diagnosis was defined according to the 2003 Rotterdam diagnosis criteria of PCOS. We included in the PCOS group women with at least two of the following criteria: (1) oligo-or anovulation; (2) clinical and/or biological hyperandrogenism; (3) polycystic morphology of ovary in ultrasound examination (at least 12 follicles with a diameter of $2-9 \mathrm{~mm}$ and/or volume $\geq 10 \mathrm{~mL}$ per ovary). ${ }^{13}$ Control subjects were healthy volunteers recruited from the community with no clinical menstrual cycle disorder and no evidence of clinical hyperandrogenism. We did not include women with any of the following conditions: pregnancy or breastfeeding, known diabetes mellitus, acute infection in the past 3 weeks, known chronic diseases (hepatic, renal, autoimmune, neoplasia, Human Immunodeficiency Virus), a very high level of hsCRP (not detectable by measuring devices), or other known hyperandrogenemia conditions (late-onset congenital adrenal hyperplasia, Cushing syndrome, and androgen secreting tumors). In addition, we ensured that oral contraceptives or drugs that could affect hormonal and metabolic profiles were discontinued at least 3 months before inclusion in the study.

\section{Procedure}

Patients with PCOS were identified from registers of the outpatient and the imaging departments and were subsequently contacted and invited to participate in the study. Control subjects were recruited amongst hospital visitors and by invitation through posters posted on social networks. After obtaining informed consent and checking the selection criteria, anthropometric and clinical data were collected. Blood samples were collected in the morning after 12 hours of overnight fasting. Plasma glucose measurement was performed immediately by God-PAP enzymatic colorimetric method (BIOLABO ${ }^{\circledR}$ kit, Paris, France), with a lower limit of detection of $0.10 \mathrm{~g} / \mathrm{L}$, an intra-assay coefficient of variation (C.V) between 1.3$0.67 \%$ for normal-high rate, and an inter-assay C.V between 1.2-1.06 for normal-high rate. C peptide and IL6 levels were measured by indirect sandwich Enzyme Linked Immuno-Sorbent Assay (ELISA) method (ELABSCIENCE $^{\circledR}$ kit, Houston, TX), with a lower limit of detection of $7.81 \mathrm{pg} / \mathrm{mL}$. C peptide had an intra-assay C.V between $4.4-5.54 \%$, and an inter-assay C.V between 4.52-5.58, whereas the intra-assay C.V of IL6 was between $3.71-6.72 \%$, and inter-assay C.V between 3.396.61. High sensitivity CRP level was evaluated by a direct immunofluorescence method (Ichroma ${ }^{\mathrm{TM}}$ kit, Boditech Med Inc, Chuncheon, Gangwon, South Korea), with a lower limit of detection of $0.5 \mathrm{mg} / \mathrm{L}$. For intra-Lot Precision, C.V is $\leq 15 \%$, and for inter-Lot Precision, C.V is $\leq 15 \%$. We used the Homeostasis Model Assessment of Insulin Resistance (HOMA-IR) index to assess insulin resistance, which was measured by multiplying fasting plasma glucose $(\mathrm{mmol} / \mathrm{L})$ by fasting serum insulin (mUI/ L) divided by 22.5 , where the insulin level was replaced by the $\mathrm{C}$ peptide. ${ }^{14}$

\section{Statistical Analysis}

Data were analyzed using Statistical Package for Social Sciences Version 23.0 (IBM SPSS Statistics for Windows, Version 23.0. Armonk, NY: IBM Corp). Continuous variables were summarized as mean \pm Standard Deviation (SD) or median [25th-75th percentiles], for normally distributed and skewed quantitative data, respectively, while categorical variables were reported as counts and percentages. The Chi-square test was used to study the association between categorical variables. We used the Student's $T$-test and non-parametric tests (Mann-Whitney $U$-test, Kruskal-Wallis test) for group's comparison of quantitative variables. Spearman correlation test was used to 
assess the linear association between skewed quantitative data. A $p$-value less than 0.05 was considered statistically significant. The minimal size for the study was calculated using the formula of Charan and Biswas with $\alpha=5 \%$ and $\beta=10 \% .{ }^{15}$ We used data from the study of $\mathrm{Hu}$ et $\mathrm{al}^{16}$ in 2011 in China, with CRP values of $1.9 \pm 2.0 \mathrm{mg} / \mathrm{L}$ for PCOS women versus $0.9 \pm 0.8 \mathrm{mg} / \mathrm{L}$ for healthy women.

\section{Results}

\section{Participants' Characteristics}

We enrolled 32 women with PCOS with a mean \pm SD age of $26.69 \pm 4.69$ years and a mean BMI of $28.94 \pm 7.78 \mathrm{Kg} /$ $\mathrm{m}^{2}$. About a third of the women were overweight $\left(\mathrm{BMI}=25-29.9 \mathrm{Kg} / \mathrm{m}^{2}\right)$ and $37.4 \%$ were obese $(\mathrm{BMI}>30$ $\mathrm{Kg} / \mathrm{m}^{2}$ ), with the same proportions in both groups. Regarding the phenotypes of PCOS women, 50\% (16/32) had phenotype A (Dysovulation | Hyperandrogenism | Polycystic ovaries), $18.75 \% \quad(6 / 32)$ phenotype B (Dysovulation | Hyperandrogenism), 9.38\% (3/32) phenotype C (Hyperandrogenism | Polycystic ovaries), and 21.88\% (7/32) phenotype D (Dysovulation | Polycystic ovaries). Women with PCOS presented a higher waist-tohip ratio (WHR) compared to control subjects $(0.93 \pm 0.12$ vs. $0.82 \pm 0.09 ; p<0.001)$. The clinical characteristics of participants are shown in Table 1.

\section{Insulin Resistance and Inflammation in Women with PCOS and Control Subjects} Women with PCOS had higher $\mathrm{C}$ peptide levels $(p=0.02)$ and HOMA-IR $(p=0.03)$ compared to controls, as shown in Table 1. Similarly, their hsCRP level was higher than that of controls $(0.63[0.32-3.81] \mathrm{mg} / \mathrm{L}$ vs. $0.47[0.15$ $1.04 \mathrm{Jg} / \mathrm{L} ; p=0.01)$, while IL-6 levels were similar between the two groups $(8.61[4.1-33.79] \mathrm{pg} / \mathrm{mL} v s .8 .80$
[5.28-38.85] $\mathrm{pg} / \mathrm{mL} ; p=0.51)$. There was no difference in levels of inflammatory markers and insulin resistance by BMI, in women with PCOS, as shown in Table 2.

A significant correlation was found between hsCRP level and BMI in the control group ( $\mathrm{r}=0.67 ; p<0.001)$, but not in women with $\operatorname{PCOS~}(\mathrm{r}=0.23 ; p=0.25)$. In women with PCOS and Controls, there was no significant linear correlation observed between the serum level of inflammatory markers and the HOMA-IR index, as shown in Table 3. HsCRP level was significantly correlated with IL-6 in both groups ( $\mathrm{r}=0.38 ; p=0.03$ for PCOS; $\mathrm{r}=0.56 ; p=0.001$ for controls). Furthermore, as shown in Table 4, no other association with serum hsCRP level was observed in the PCOS group.

\section{Discussion}

We aimed to study inflammatory markers and insulin resistance in a population of PCOS patients compared to their age and BMI matched controls. We found that the level of hsCRP was higher in women with PCOS compared to controls. There was no difference in IL-6 levels between both groups, but IL-6 was correlated to hsCRP. The HOMA-IR index was higher in women with PCOS compared to controls. In addition, no factor associated with hsCRP level in women with PCOS was identified.

The higher level of hsCRP in women with PCOS compared to controls supports the conclusions of several studies in different populations of women with PCOS, including a recent report of Rudnicka et $\mathrm{al}^{9}$ suggesting the presence of a low-grade chronic inflammation in PCOS. ${ }^{17,18}$ Indeed, hyperandrogenism in women with PCOS seems to lead to adipocyte hypertrophy, which activates the nuclear factor kappa-B (NF-kB). Activation of this factor induces the release of several inflammatory cytokines, which in turn

Table I Clinical Characteristics and Insulin Resistance Parameters of Participants

\begin{tabular}{|l|l|l|l|}
\hline & PCOS $(\mathbf{n}=\mathbf{3 2})^{*}$ & Controls $(\mathbf{n = 3 2})^{*}$ & -value \\
\hline Age (years) & $26.69 \pm 4.69$ & $27.09 \pm 4.98$ & 0.74 \\
BMI $\left(\mathrm{Kg} / \mathrm{m}^{2}\right)$ & $28.94 \pm 7.78$ & $28.29 \pm 6.96$ & 0.72 \\
WHR & $0.93 \pm 0.12$ & $0.82 \pm 0.09$ & $<0.001$ \\
SBP $(\mathrm{mmHg})$ & $118.31 \pm 11.78$ & $117.75 \pm 11.53$ & 0.85 \\
DBP $(\mathrm{mmHg})$ & $77.19 \pm 10.37$ & $79.13 \pm 8.35$ & 0.41 \\
Glycemia (mmol/L) & $5.27 \pm 1.05$ & $5.35 \pm 0.94$ & 0.76 \\
C peptide (mUl/L)) & $4.98 \pm 3.83$ & $3.25 \pm 1.62$ & $\mathbf{0 . 0 2}$ \\
HOMA-IR & $1.15 \pm 0.90$ & $0.77 \pm 0.38$ & $\mathbf{0 . 0 3}$ \\
\hline
\end{tabular}

Notes: *Data are presented as Mean $\pm \mathrm{SD}$; text in bold represents significant values.

Abbreviations: BMI, body mass index; WHR, waist-to-hip ratio; SBP, systolic blood pressure; DBP, diastolic blood pressure; HOMA-IR, homeostasis model assessment of insulin resistance. 
Table 2 Inflammatory Markers and Insulin Resistance According to BMI

\begin{tabular}{|l|l|l|l|l|}
\hline & Normal* & Overweight* & Obese* & $p$-value \\
\hline PCOS & & & & \\
HsCRP $(\mathrm{mg} / \mathrm{L})$ & $0.52[0.22-3.13]$ & $0.80[0.30-2.93]$ & $2.63[0.35-4.26]$ & $32.94[7.04-55.74]$ \\
IL6 (pg/mL) & $5.48[2.35-9.79]$ & $8.61[3.71-20.00]$ & $1.12[0.52-2.48]$ & 0.53 \\
HOMA-IR & $0.55[0.38-0.65]$ & $0.98[0.67-1.79]$ & & 0.19 \\
\hline Controls & & & $1.05[0.42-1.40]$ \\
HsCRP $(\mathrm{mg} / \mathrm{L})$ & $0.16[0.10-0.53]$ & $0.52[0.27-0.99]$ & $34.62[9.39-57.43]$ & 0.06 \\
IL6 (pg/mL) & $4.69[2.94-9.59]$ & $10.77[5.77-40.12]$ & $0.64[0.37-0.88]$ & $\mathbf{0 . 0 3}$ \\
HOMA-IR & $0.71[0.30-1.15]$ & $0.91[0.56-1.08]$ & 0.62 \\
\hline
\end{tabular}

Notes: * Data are presented as median [25th-75th percentile]; text in bold represents significant values.

Abbreviations: HsCRP, high sensitivity $\mathrm{C}$ reactive protein; IL6, interleukin 6; HOMA-IR, homeostasis model assessment of insulin resistance.

Table 3 Correlation between Inflammatory Markers and HOMA-IR Index in Both Groups

\begin{tabular}{|l|l|l|}
\hline & $\begin{array}{l}\text { Spearman } \\
\text { Correlation (r) }\end{array}$ & p-value \\
\hline HsCRP & & \\
PCOS & 0.10 & 0.62 \\
Controls & 0.12 & 0.55 \\
\hline IL6 & & \\
PCOS & -0.14 & 0.49 \\
Controls & 0.16 & 0.43 \\
\hline
\end{tabular}

Abbreviations: $\mathrm{HsCRP}_{\mathrm{s}}$ high sensitivity C reactive protein; IL6, interleukin 6. stimulate the production of CRP by the liver. ${ }^{19,20}$ On the other hand, we did not observe any difference in IL-6 levels in the PCOS group compared to the controls. These findings are similar to previous reports on IL-6. ${ }^{17,21}$ IL- 6 is known to stimulate the liver's production of CRP. ${ }^{22}$ The difference observed for hsCRP, but not for IL-6 in the two groups, can be explained by the fact that, beside IL-6, other mediators can stimulate CRP production. ${ }^{22}$ Together, our data suggest that there is a low-grade inflammation in sub-Saharan African women with PCOS. They are therefore predisposed to cardiometabolic complications, and it would be interesting to

Table 4 Association between Clinical and Biological Characteristics of Women with PCOS and the hsCRP Level

\begin{tabular}{|c|c|c|c|c|}
\hline \multirow[t]{2}{*}{ Variables $(n=26)$} & \multicolumn{2}{|c|}{ HsCRP } & \multirow[t]{2}{*}{ OR (95\% IC) } & \multirow[t]{2}{*}{$P$-value } \\
\hline & High & Low & & \\
\hline \multicolumn{5}{|l|}{ Age (years) } \\
\hline$<25$ & $7(53.8)$ & $6(46.2)$ & $1.3(0.29-6.36)$ & 0.69 \\
\hline$\geq 25$ & $6(46.2)$ & $7(53.8)$ & & \\
\hline \multicolumn{5}{|l|}{ BMI } \\
\hline Obese & $4(57.1)$ & $3(42.9)$ & $1.48(0.25-8.49)$ & I \\
\hline Non-obese & $9(47.4)$ & $10(52.6)$ & & \\
\hline \multicolumn{5}{|l|}{ WHR } \\
\hline$>0.80$ & II (52.4) & $10(47.6)$ & $0.65(0.22-11.99)$ & I \\
\hline$\leq 0.80$ & $2(40.0)$ & $3(60.0)$ & & \\
\hline \multicolumn{5}{|c|}{ Hyperandrogenism } \\
\hline Yes & $10(52.6)$ & $9(47.4)$ & $1.48(0.26-8.50)$ & I \\
\hline No & $3(42.9)$ & $4(57.1)$ & & \\
\hline \multicolumn{5}{|l|}{ HOMA-IR } \\
\hline$\geq 0.72$ & $8(57.1)$ & $6(42.9)$ & $1.86(0.39-8.89)$ & 0.69 \\
\hline$<0.72$ & $5(4 \mid .7)$ & $7(58.3)$ & & \\
\hline
\end{tabular}

Abbreviations: $\mathrm{HsCRP}$, high sensitivity $\mathrm{C}$ reactive protein; OR, odds ratio; $\mathrm{Cl}$, confidence interval; $\mathrm{BMI}$, body mass index; WHR, waist-to-hip ratio; HOMA-IR, homeostasis model assessment of insulin resistance. 
study the long-term effect of anti-inflammatory drugs on the occurrence of these.

As expected, we found a high HOMA-IR index in women with PCOS compared to their matched controls. In Cameroon, Doh et $\mathrm{al}^{23}$ in 2016, conducted a study including 14 PCOS patients on the same population and found similar results using the hyperinsulinemic euglycemic clamp. Insulin resistance is associated with an increased secretion of insulin. Physiologically, there is a potential relation between hyperinsulinism and hyperandrogenism. Indeed, insulin resistance results in a compensatory increase in insulin secretion in order to maintain normal glucose homeostasis. ${ }^{24}$ This hyperinsulinism stimulates the secretion of luteinizing hormone by the gonadotropic cells of the anterior pituitary gland, and potentiates its action on the internal thecal cells of the ovary for the production of androgens. ${ }^{25}$ In addition, hyperinsulinism is responsible for premature sensitivity of follicular cells to luteinizing hormone, altering the selection of the dominant follicle-by-follicle stimulating hormone. ${ }^{26}$ Hyperandrogenism and ovulation disorders are the main characteristics of PCOS. ${ }^{2}$

In our study we found that WHR is higher in women with PCOS than in controls. One study showed that abdominal obesity causes additional disturbances of metabolic and hormonal parameters in PCOS. ${ }^{27}$ Furthermore, visceral obesity seems to also be associated with chronic low-grade inflammation. ${ }^{28}$ One may wonder whether the high level of hsCRP and insulin resistance found in our study would be related to this difference in WHR? However, we did not find any association between WHR and hsCRP level in our study.

We did not find any factors associated with the hsCRP level in women with PCOS. With regard to the correlation between hsCRP and BMI, it should be noted that we looked for a correlation between these two variables in each group. We found a strong positive correlation in the control group, but not in patients with PCOS. This suggests that once women have developed PCOS with its inflammatory state, obesity becomes a minor determinant factor of inflammation, probably because of the interplay of many additional factors associated with PCOS. No correlation was found between hsCRP level and HOMAIR, and there was no association between the hsCRP level and various clinical parameters. These results are opposed to those of most previous studies on the same population.9,16 This difference could be due to different population backgrounds. None of the studies reporting an association between hsCRP and HOMA-IR were done in sub-Saharan African populations. In addition, there are also some studies that did not report any correlation between hsCRP level and HOMA-IR Index, suggesting that this might indeed be study- or population-dependent. ${ }^{29,30}$ Can the inflammation be due to another cause? Studies suggested an association between the presence of low-grade chronic inflammation and infections, such as Chlamydia trachomatis or Helicobacter pylori, which are highly prevalent in our context. ${ }^{31-33}$ Future studies could explore the presence of these infections in PCOS women, with low grade chronic inflammation, especially Chlamydia trachomatis infection, known to be frequent and to be strongly associated with infertility. ${ }^{34}$

\section{Strength and Limitations}

As a strength, we matched patients and controls for age and BMI, which are confounding factors for the occurrence of insulin resistance and inflammation. ${ }^{35,36}$ As limitations, prior to inclusion, we did not carry out any biological examinations for acute or chronic inflammation. However, women who had high levels of inflammatory markers - suggestive of any acute inflammation - measured within the framework of the study, were excluded from the analysis. The reference test for determining the level of insulin resistance is the hyperinsulinemic euglycemic clamp. ${ }^{37}$ It is laborious and expensive to carry out and requires an average of 2-3 hours, hence its use is reserved for a few research studies. In our study, we used an index obtained on a fasting sample, notably the HOMA-IR index, which has a very strong correlation with the reference test $(\mathrm{r}=0.88){ }^{38}$

\section{Conclusion}

Patients with PCOS have low grade chronic inflammation represented by higher levels of hsCRP, and a higher level of insulin resistance compared to controls. However, obesity and the specific characteristics of PCOS are not associated with this inflammation. Identification of factors associated with this low-grade chronic inflammation in PCOS, like the presence of some pathogens, requires further study.

\section{Abbreviations}

BMI, body mass index; C.V, coefficient of variation; DBP, diastolic blood pressure; HOMA-IR, homeostasis model assessment of insulin resistance; hsCRP, high sensitivity $\mathrm{C}$ reactive protein; IL-6, interleukin 6; PCOS, polycystic 
ovary syndrome; SBP, systolic blood pressure; SD, standard deviation; WHR, waist-to-hip ratio.

\section{Data Sharing Statement}

The data used to support the findings of this study are available from the corresponding author upon request.

\section{Ethics Approval and Informed Consent}

The Regional Ethics Committee approved this study for Human Health Research of Centre Region, Cameroon (CE $\mathrm{N}^{\circ}$ 0614/CRERSHC/2020). All participants provided written informed consent before inclusion and the study was conducted in accordance with principles of biomedical research as stated in the Declaration of Helsinki. ${ }^{39}$

\section{Acknowledgments}

We would like to thank the staff of the laboratory of the National Obesity Center of the central hospital of Yaoundé for carrying out the biological analyses. We gratefully acknowledge all the patients who agreed to take part in this study. We would also like to thank Floriane Djapa Tofeun and Serge Armand Guifo Foubi for their help during data collection, and Magellan Guewo-Fokeng and Esther Astrid Mbono Samba for proofreading.

\section{Author Contributions}

All authors contributed to the data analysis, drafting, or revising of the article, have agreed on the journal to which the article will be submitted, gave final approval of the version to be published, and agree to be accountable for all aspects of the work.

\section{Funding}

This study was funded by the first three authors.

\section{Disclosure}

The authors report no conflicts of interest in this work.

\section{References}

1. Khan KA, Stas S, Kurukulasuriya LR. Polycystic ovarian syndrome. J Cardiometab Syndr. 2006;1(2):125-130. doi:10.1111/j.15594564.2006.05675.x

2. Bozdag G, Mumusoglu S, Zengin D, Karabulut E, Yildiz BO. The prevalence and phenotypic features of polycystic ovary syndrome: a systematic review and meta-analysis. Hum Reprod. 2016;31(12):28412855. doi:10.1093/humrep/dew218

3. Azziz R, Adashi EY. Stein and Leventhal: 80 years on. Am J Obstet Gynecol. 2016;214(2):247-e1.
4. Cussons AJ, Watts GF, Burke V, Shaw JE, Zimmet PZ, Stuckey BGA. Cardiometabolic risk in polycystic ovary syndrome: a comparison of different approaches to defining the metabolic syndrome. Hum Reprod. 2008;23(10):2352-2358. doi:10.1093/humrep/den263

5. Azziz R, Carmina E, Dewailly D, et al. The androgen excess and PCOS society criteria for the polycystic ovary syndrome: the complete task force report. Fertil Steril. 2009;91(2):456-488.

6. Popovic M, Sartorius G, Christ-Crain M. Chronic low-grade inflammation in polycystic ovary syndrome: is there a (patho)-physiological role for interleukin-1? Semin Immunopathol. 2019;41(4):447-459. doi:10.1007/s00281-019-00737-4

7. González F. Inflammation in polycystic ovary syndrome: underpinning of insulin resistance and ovarian dysfunction. Steroids. 2012;77 (4):300-305. doi:10.1016/j.steroids.2011.12.003

8. Sharma P. Inflammation and the metabolic syndrome. Indian J Clin Biochem. 2011;26(4):317-318. doi:10.1007/s12291-011-0175-6

9. Rudnicka E, Kunicki M, Suchta K, Machura P, Grymowicz M, Smolarczyk R. Inflammatory markers in women with polycystic ovary syndrome. Biomed Res Int. 2020;2020:1-10. doi:10.1155/ 2020/4092470

10. Duleba AJ, Dokras A. Is PCOS an inflammatory process? Fertil Steril. 2012;97(1):7-12. doi:10.1016/j.fertnstert.2011.11.023

11. Koppalli S, Vivekananda RB, Vijayaraghavan R, Paluru R, Rangarao T, Sarma SSB. Evaluation of serum hs-CRP concentrations in reproductive women with polycystic ovarian syndrome (PCOS). Biomed Res. 2017;28(5).

12. Kelly CCJ, Lyall H, Petrie JR, Gould GW, Connell JMC, Sattar N. Low grade chronic inflammation in women with polycystic ovarian syndrome. J Clin Endocrinol Metab. 2001;86(6):2453-2455. doi: $10.1210 /$ jcem. 86.6 .7580

13. Rotterdam ESHRE/ASRM-Sponsored PCOS Consensus Workshop Group. Revised 2003 consensus on diagnostic criteria and longterm health risks related to polycystic ovary syndrome. Fertil Steril. 2004;81(1):19-25. doi:10.1016/j.fertnstert.2003.10.004

14. Pacini G, Mari A. Methods for clinical assessment of insulin sensitivity and $\beta$-cell function. Best Pract Res Clin Endocrinol Metab. 2003;17(3):305-322. doi:10.1016/S1521-690X(03)00042-3

15. Charan J, Biswas T. How to calculate sample size for different study designs in medical research? Indian J Psychol Med. 2013;35(2):121126. doi:10.4103/0253-7176.116232

16. Hu W, Qiao J, Yang Y, Wang L, Li R. Elevated C-reactive protein and monocyte chemoattractant protein-1 in patients with polycystic ovary syndrome. Eur J Obstet Gynecol Reprod Biol. 2011;157(1):53-56. doi:10.1016/j.ejogrb.2011.03.015

17. Escobar-Morreale HF, Luque-Ramírez M, González F. Circulating inflammatory markers in polycystic ovary syndrome: a systematic review and metaanalysis. Fertil Steril. 2010;95(3):1048-1058.

18. Boulman N, Levy Y, Leiba R, et al. Increased C-reactive protein levels in the polycystic ovary syndrome: a marker of cardiovascular disease. $J$ Clin Endocrinol Metab. 2004;89(5):2160-2165. doi:10.1210/jc.2003-031096

19. Wisse BE. The inflammatory syndrome: the role of adipose tissue cytokines in metabolic disorders linked to obesity. J Am Soc Nephrol. 2004;15(11):2792-2800. doi:10.1097/01.ASN.0000141966.69934.21

20. Spritzer PM, Lecke SB, Satler F, Morsch DM. Adipose tissue dysfunction, adipokines, and low-grade chronic inflammation in polycystic ovary syndrome. Reproduction. 2015;149(5):R219-R227. doi:10.1530/REP-14-0435

21. Souza Dos Santos AC, Soares NP, Costa EC, de Sá JCF, Azevedo GD, Lemos TMAM. The impact of body mass on inflammatory markers and insulin resistance in polycystic ovary syndrome. Gynecol Endocrinol. 2015;31(3):225-228. doi:10.3109/09513590.2 014.976546

22. Black S, Kushner I, Samols D. C-reactive protein. J Biol Chem. 2004;279(47):48487-48490. doi:10.1074/jbc.R400025200 
23. Doh E, Mbanya A, Kemfang-Ngowa JD, et al. The relationship between adiposity and insulin sensitivity in African women living with the polycystic ovarian syndrome: a Clamp Study. Int J Endocrinol. 2016;2016:1-6. doi:10.1155/2016/9201701

24. Dunaif A. Insulin resistance and the polycystic ovary syndrome: mechanism and implications for pathogenesis. Endocr Rev. 1997;18 (6):774-800.

25. Nestler JE, Jakubowicz DJ, de Vargas AF, Brik C, Quintero N, Medina F. Insulin stimulates testosterone biosynthesis by human thecal cells from women with polycystic ovary syndrome by activating its own receptor and using inositolglycan mediators as the signal transduction system. J Clin Endocrinol Metab. 1998;83(6):20012005.

26. Willis DS, Watson H, Mason HD, Galea R, Brincat M, Franks S. Premature response to luteinizing hormone of granulosa cells from anovulatory women with polycystic ovary syndrome: relevance to mechanism of anovulation. None. 1998;83(11):3984-3991.

27. Franik G, Bizoń A, Włoch S, et al. The effect of abdominal obesity in patients with polycystic ovary syndrome on metabolic parameters Eur Rev Med Pharmacol Sci. 2017;21(21):4755-4761.

28. Lisko I, Tiainen K, Stenholm S, et al. Inflammation, adiposity, and mortality in the oldest old. Rejuvenation Res. 2012;15(5):445-452. doi:10.1089/rej.2011.1310

29. Goswami S, Choudhuri S, Bhattacharya B, et al. Chronic inflammation in polycystic ovary syndrome: a case-control study using multiple markers. Int J Reprod Biomed. 2021;19(4):313-320.

30. Ganie MA, Hassan S, Nisar S, et al. High-sensitivity C-reactive protein (hs-CRP) levels and its relationship with components of polycystic ovary syndrome in Indian adolescent women with polycystic ovary syndrome (PCOS). Gynecol Endocrinol. 2014;30 (11):781-784. doi:10.3109/09513590.2014.924099

31. Yavasoglu I, Kucuk M, Cildag B, Arslan E, Gok M, Kafkas S. A novel association between polycystic ovary syndrome and Helicobacter pylori. Am J Med Sci. 2009;338(3):174-177. doi:10.1097/MAJ.0b013e3181a63c8a
32. Morin-Papunen LC, Duleba AJ, Bloigu A, Järvelin M-R, Saikku P, Pouta A. Chlamydia antibodies and self-reported symptoms of oligoamenorrhea and hirsutism: a new etiologic factor in polycystic ovary syndrome? Fertil Steril. 2010;94(5):1799-1804. doi:10.1016/j. fertnstert.2009.10.021

33. Dursun E, Akalın FA, Güncü GN, et al. Periodontal disease in polycystic ovary syndrome. Fertil Steril. 2011;95(1):320-323. doi:10.1016/j.fertnstert.2010.07.1052

34. Essome MCN, Nsawir BJ, Nana RD, Molu P, Mohamadou M. Etude séro-epidémiologique de trois infections sexuellement transmissibles (Chlamydia Trachomatis, Hépatite B, Syphilis): cas de 1'Hôpital de District de Nkoldongo à Yaoundé. Pan Afr Med J. 2016;25:244. doi:10.11604/pamj.2016.25.244.11107

35. Milan-Mattos JC, Anibal FF, Perseguini NM, et al. Effects of natural aging and gender on pro-inflammatory markers. Braz J Med Biol Res. 2019;52(9). doi:10.1590/1414-431x20198392

36. Monteiro R, Azevedo I. Chronic inflammation in obesity and the metabolic syndrome. Mediators Inflamm. 2010;2010:1-10. doi:10.1155/2010/289645

37. Wallace TM, Matthews DR. The assessment of insulin resistance in man. Diabet Med. 2002;19(7):527-534. doi:10.1046/j.14645491.2002.00745.x

38. Matthews DR, Hosker JP, Rudenski AS, Naylor BA, Treacher DF, Turner RC. Homeostasis model assessment: insulin resistance and beta-cell function from fasting plasma glucose and insulin concentrations in man. Diabetologia. 1985;28(7):412-419. doi:10.1007/BF00280883

39. Goodyear MDE, Krleza-Jeric K, Lemmens T. The declaration of Helsinki. BMJ. 2007;335(7621):624-625. doi:10.1136/bmj.39339.610000.BE
Journal of Inflammation Research

\section{Publish your work in this journal}

The Journal of Inflammation Research is an international, peerreviewed open-access journal that welcomes laboratory and clinical findings on the molecular basis, cell biology and pharmacology of inflammation including original research, reviews, symposium reports, hypothesis formation and commentaries on: acute/chronic inflammation; mediators of inflammation; cellular processes; molecular mechanisms; pharmacology and novel anti-inflammatory drugs; clinical conditions involving inflammation. The manuscript management system is completely online and includes a very quick and fair peerreview system. Visit http://www.dovepress.com/testimonials.php to read real quotes from published authors. 